\title{
Large-time behavior of solutions to the inflow problem of the non-isentropic micropolar fluid model
}

\author{
Haibo Cui ${ }^{1}$ and Junpei Gao ${ }^{1}$ \\ ${ }^{1}$ Huaqiao University
}

May 5, 2020

\begin{abstract}
We investigate the asymptotic behavior of solutions to the initial boundary value problem for the micropolar fluid model in a half line $\$ \backslash R_{-}\{+\}:=(0, \backslash$ infty $) . \$$ Inspired by the relationship between micropolar fluid and Navier-Stokes, we prove that the composite wave onsisting of the transonic boundary layer solution, the 1-rarefaction wave, the viscous 2-contact wave and the 3-rarefaction wave for the inflow problem on the micropolar fluid model is time-asymptotically stable under some smallness conditions. Meanwhile, we obtain the global existence of solutions based on the basic energy method.
\end{abstract}

\section{Hosted file}

\begin\{CJK\}\{UTF8\}\{gbsn\}. \end\{CJK\}\selectlanguage\{english\}pdf available at https://authorea. } com/users/300836/articles/430567-large-time-behavior-of-solutions-to-the-inflow-problemof-the-non-isentropic-micropolar-fluid-model 